VOL. 60 (1999) [479-482]

\title{
PLANE CURVES AND p-ADIC ROOTS OF UNITY
}

\author{
José FeLIPE VolOCH
}

\begin{abstract}
We prove the following result: Let $f(x, y)$ be a polynomial of degree $d$ in two variables whose coefficents are integers in an unramified extension of $\mathbf{Q}_{p}$. Assume that the reduction of $f$ modulo $p$ is irreducible of degree $d$ and not a binomial. Assume also that $p>d^{2}+2$. Then the number of solutions of the inequality $\left|f\left(\zeta_{1}, \zeta_{2}\right)\right|<p^{-1}$, with $\zeta_{1}, \zeta_{2}$ roots of unity in $\overline{\mathbf{Q}}_{p}$ or zero, is at most $p d^{2}$.
\end{abstract}

Let $\mathbf{C}_{p}$ be the completion of the algebraic closure of $\mathbf{Q}_{p}$ with its usual norm extending that of $\mathbf{Q}_{p}$. In [5], a result which implies the following statement was proved. If $f(x, y) \in \mathbf{C}_{p}[x, y]$ there exists a positive constant $c$ such that, for any roots of unity $\zeta_{1}, \zeta_{2}$, either $f\left(\zeta_{1}, \zeta_{2}\right)=0$ or $\left|f\left(\zeta_{1}, \zeta_{2}\right)\right| \geqslant c$. (A similar result holds for polynomials with an arbitrary number of variables.) In general, however, there is little information about the value of $c$. In the case that $f$ is linear and its coefficients are units in an unramified extension of $\mathbf{Q}_{p}$, it was proved in [5] that the inequality $\left|f\left(\zeta_{1}, \zeta_{2}\right)\right| \leqslant p^{-2}$ had at most $p$ solutions $\zeta_{1}, \zeta_{2}$ roots of unity or zero. The purpose of this note is to obtain a similar result for more general polynomials in two variables. Recall that a binomial is a polynomial with (at most) two non-zero coefficients. Our main result is then:

THEOREM . Let $f(x, y)$ be a polynomial of degree $d$ in two variables whose coefficents are integers in an unramified extension of $\mathbf{Q}_{p}$. Assume that the reduction of $f$ modulo $p$ is irreducible of degree $d$ and not a binomial. Assume also that $p>d^{2}+2$. Then the number of solutions of the inequality $\left|f\left(\zeta_{1}, \zeta_{2}\right)\right|<p^{-1}$, with $\zeta_{1}, \zeta_{2}$ roots of unity in $\overline{\mathbf{Q}}_{p}$ or zero, is at most $p d^{2}$.

Proof: We shall first prove the theorem under the additional condition that we are dealing with roots of unity of order prime to $p$. The inequality then translates into $f\left(\zeta_{1}, \zeta_{2}\right) \equiv 0\left(\bmod p^{2}\right)$. The ring of integers of the completion of the maximal unramified extension of $Q_{p}$ can be viewed as the ring of Witt vectors over the algebraic closure of $F_{p}$ and, since we are interested only in the situation modulo $p^{2}$, we can work in the Witt vectors of length two over the algebraic closure of $\mathbf{F}_{p}$. We are thus interested

Received 3rd March, 1999

The author would like to thank the TARP (grant \#ARP-0006) and the NSA (grant MDA904-97-1-0038) for financial support.

Copyright Clearance Centre, Inc. Serial-fee code: 0004-9727/99 \$A2.00+0.00. 
in the solutions of the equation $f((x, 0),(y, 0))=(0,0)$. This equation translates into the system $f_{0}(x, y)=g(x, y)=0$, where $f_{0}$ is the reduction of $f$ modulo $p$ and the polynomial $g$ is the reduction modulo $p$ of the polynomial $\left(f^{\sigma}\left(x^{p}, y^{p}\right)-f(x, y)^{p}\right) / p$ and $\sigma$ is the Frobenius automorphism of the ring of Witt vectors. Clearly $g$ has degree at most $p d$ and, since $f_{0}$ is assumed irreducible of degree $d$, the result we want follows from Bézout's theorem unless $f_{0}$ divides $g$, which we proceed to show cannot happen.

Let $X$ be the irreducible plane curve defined by $f_{0}(x, y)=0$. We shall derive a contradiction from the assumption that $g$ vanishes identically on $X$. If $g=0$ on $X$ then, by differentiating $g(x, y)=0$ we obtain $g_{x}+g_{y} d y / d x=0$ and, from the definition of $g$ we have $g_{x}=f_{x}^{\sigma}\left(x^{p}, y^{p}\right) x^{p-1}-f(x, y)^{p-1} f_{x}=f_{0 x}^{p} x^{p-1}$ on $X$. Likewise $g_{y}=f_{0 y}^{p} y^{p-1}$ on $X$. Since $f_{0}$ is of degree less than $p$ and is not a binomial, we have that $f_{0 x}, f_{0 y}$ are non-zero. Using that $d y / d x=-f_{0 x} / f_{0 y}$, we obtain the identity $f_{0 x}^{p-1} x^{p-1}=f_{0 y}^{p-1} y^{p-1}$, on $X$. This gives $x f_{0 x}=c y f_{0 y}$ for some $c \in \mathbf{F}_{p}$. The lemma below ensures that this cannot hold under the assumptions that $p>d^{2}$ and $f_{0}$ is not a binomial and this will complete the proof in the case the roots of unity are of order prime to $p$.

If $\zeta_{1}, \zeta_{2}$ are arbitrary roots of unity satisfying the inequality $\left|f\left(\zeta_{1}, \zeta_{2}\right)\right|<p^{-1}$ we can write $\zeta_{i}=\lambda_{i} \eta_{i}, i=1,2$ where the $\lambda_{i}$ are of order prime to $p$ and the $\eta_{i}$ are of $p$-power order and are not both equal to one. We shall show that this inequality has no such solution. By a harmless change of coordinates we may assume that $\lambda_{i}=1, i=1,2$. Further, perhaps after switching $x$ and $y$ if necessary, we may assume that $\eta_{2}=\eta_{1}^{\text {r for }}$ some integer $r$. We write $\eta_{1}=1+\pi$ and notice that the inequality $\left|f\left(\zeta_{1}, \zeta_{2}\right)\right|<p^{-1}$ implies $f\left(1+\pi,(1+\pi)^{r}\right) \equiv 0\left(\bmod \pi^{p-1}\right)$. On the other hand if $\mathcal{O}$ is the ring of integers of the field $F\left(\eta_{1}\right)$, where $F$ is a unramified extension of $Q_{p}$ containing the coefficients of $f$, then $\mathcal{O} / \pi^{p-1}$ is isomorphic to $k[t] / t^{p-1}$, where $k$ is the residue field of $F$. Therefore we obtain $f_{0}\left(1+t,(1+t)^{r}\right) \equiv 0\left(\bmod t^{p-1}\right)$. This implies, with notation as above, that $y / x^{r}-1$ has a zero of order at least $p-1$ at some place of $X$ centred at $(1,1)$, so the differential $d y / y-r d x / x$ has a zero of order at least $p-2$ at that same place. However, this differential has at most $3 d$ poles counted with multiplicity, so at most $3 d+2 g-2$ zeros, where $g$ is the genus of $X$ unless it is identically zero. Now, $3 d+2 g-2 \leqslant 3 d+d(d-3)=d^{2}<p-2$, by hypothesis, so the differential is identically zero, which, using that $d y / d x=-f_{0 x} / f_{0 y}$, leads to a contradiction with the lemma below.

It remains only to prove:

Lemma . Let $f(x, y)=0$ define an irreducible plane curve $X$ of degree $d$ over an algebraically closed field $k$ of characteristic $p$ satisfying $p>d^{2}$. If $x f_{x}=c y f_{y}$ on $X$ for some $c$ in $k$ then $f$ is a binomial.

PROOF: The hypothesis means an identity $x f_{x}-c y f_{y}=b f$ for some $b$ in $k$. If 
$f(x, y)=\sum a_{i j} x^{i} y^{j}$ we get $a_{i j}(i-c j-b)=0$ for all $i, j$. Suppose first that $b=0$. For any $i, j, i^{\prime}, j^{\prime}$ with both $a_{i j}, a_{i^{\prime} j^{\prime}}$ non-zero, we get $i-c j=i^{\prime}-c j^{\prime}=0$ which implies that $i j^{\prime}-i^{\prime} j=(i-c j) j^{\prime}-\left(i^{\prime}-c j^{\prime}\right) j=0$ in $k$, which means that $p$ divides $i j^{\prime}-i^{\prime} j$, but under our assuption that $p>d^{2}$, this implies that $i j^{\prime}=i^{\prime} j$ and this implies that the value of $i / j$ is constant for all $i, j$ with $a_{i j} \neq 0$. So $f(x, y)=\sum_{r} a_{r m, r n} x^{r m} y^{r n}$ which can be written as a constant multiple of a product of terms of the form $x^{m} y^{n}-\alpha$ and, since $f$ is irreducible, we conclude that $f$ is a binomial.

Assume now that $b$ is not zero. First of all, if $f$ is a polynomial in just one variable and is irreducible, then it is a binomial and we are done. Therefore, we may assume that there exists $i_{1}, j_{1}$ with $a_{0 j_{1}}, a_{i_{1} 0}$ both non-zero and we get that $i_{1}=b$ and $c j_{1}=-b$, so $c$ is not zero and $c=-i_{1} / j_{1}$. If $i, j$ are such that $a_{i j} \neq 0$ then $i+j i_{1} / j_{1}-i_{1}=0$ in $k$ so $i j_{1}+j i_{1} \equiv i_{1} j_{1}(\bmod p)$. But $i_{1}, j_{1} \leqslant d, i+j \leqslant d$, therefore $0 \leqslant i j_{1}+j i_{1}, i_{1} j_{1} \leqslant$ $d^{2}<p$ so $i j_{1}+j i_{1}=i_{1} j_{1}$. Let $\delta=\left(i_{1}, j_{1}\right), i_{1}=m \delta, j_{1}=n \delta,(m, n)=1$. We get $i n+j m=m n \delta$, so $m|i, n| j$ and writing $i=m u, j=m v$ we get $u+v=\delta$. Thus $f(x, y)=\sum_{u} a_{m u, n(\delta-u)} x^{m u} y^{n(\delta-u)}$ which can be written as a constant multiple of a product of terms of the form $x^{m}-\alpha y^{n}$ and, since $f$ is irreducible, we conclude that $f$ is a binomial.

REMARKs. (i) If $X$ is a projective curve of genus bigger than one embedded in an Abelian variety $A$, all defined over an unramified extension of $\mathrm{Q}_{p}$, then Raynaud [4] proved that there are only finitely many torsion points of $A$ of order prime to $p$ which are in $X$ modulo $p^{2}$ and Buium [1] gave an explicit bound for the number of those points. Perhaps the techniques of Coleman [2] could be used to extend this result to the full torsion and obtain an Abelian analogue of the above result.

(ii) A special case of Lang's extension of the Manin-Mumford conjecture, proved by Ihara, Serre and Tate (see [3, Chapter 8, Theorem 6.1]) states that if $f(x, y)$ is an irreducible polynomial, not a binomial, over a field of characteristic zero, then there are only finitely many roots of unity $\zeta_{1}, \zeta_{2}$ with $f\left(\zeta_{1}, \zeta_{2}\right)=0$. This follows from the above theorem by choosing $p$ large enough such that the field generated by the coefficents of $f$ embeds in $\mathbf{Q}_{p}$ and such that the hypotheses of the theorem hold.

\section{REFERENCES}

[1] A. Buium, 'Geometry of p-jets', Duke Math. J. 82 (1996), 349-367.

[2] R.F. Coleman, 'Ramified torsion points on curves', Duke Math. J. 54 (1987), 615-640.

[3] S. Lang, Fundamentals of diophantine geometry (Springer-Verlag, Berlin, Heidelberg, New York, 1983).

[4] M. Raynaud, 'Courbes sur une variété abélienne et points de torsion', Invent. Math. 71 (1983), 207-233. 
[5] J. Tate and J.F. Voloch, 'Linear forms in p-adic roots of unity', Internat. Math. Res. Notices 12 (1996), 589-601.

Department of Mathematics

University of Texas

Austin TX 78712

United States of America

e-mail: voloch@math.utexas.edu 